ARMA manuscript No.

(will be inserted by the editor)

\title{
Convex Four Body Central Configurations with Some Equal Masses
}

\author{
Ernesto Perez-Chavela and Manuele Santoprete
}

\begin{abstract}
We prove that there is a unique convex non-collinear central configuration of the planar Newtonian four-body problem when two equal masses are located at opposite vertices of a quadrilateral and, at most, only one of the remaining masses is larger than the equal masses. Such central configuration posses a symmetry line and it is a kite shaped quadrilateral. We also show that there is exactly one convex non-collinear central configuration when the opposite masses are equal. Such central configuration also posses a symmetry line and it is a rhombus.
\end{abstract}

\section{Introduction}

The Newtonian planar $n$-body problem is the study of the dynamics of $n$ point particles with masses $m_{i} \in \mathbb{R}^{+}$and positions $q_{i} \in \mathbb{R}^{2}(i=1, \ldots, n)$, moving according to Newton's laws of motion:

$$
m_{i} \ddot{q}_{i}=\frac{\partial U}{\partial q_{i}}
$$

where $U(q)$ is the Newtonian potential

$$
U(q)=\sum_{i<j} \frac{m_{i} m_{j}}{r_{i j}}
$$

where $r_{i j}=\left\|q_{i}-q_{j}\right\|$. Let $q=\left(q_{1}, \ldots, q_{n}\right) \in \mathbb{R}^{2 n}$ and let $M$ be the matrix $\operatorname{diag}\left[m_{1}, m_{1}, m_{2}, m_{2} \ldots, m_{n}, m_{n}\right]$, then the equations of motion can be written as follows:

$$
\ddot{q}=M^{-1} \frac{\partial U}{\partial q} .
$$


To study this problem, without any loss of generality, we can assume the center of mass is fixed at the origin and consider the space

$$
\Omega_{n}=\left\{q=\left(q_{1}, q_{2}, \ldots, q_{n}\right) \in \mathbb{R}^{2 n} \mid \sum_{i=i}^{n} m_{i} q_{i}=0\right\} .
$$

Because the potential is singular when two particles have the same position it is natural to assume that the configuration avoids the set $\Delta=\bigcup_{i \leq j} \Delta_{i j}$ where

$$
\Delta_{i j}=\left\{\left(q_{1}, q_{2}, \ldots, q_{n}\right) \in \mathbb{R}^{2 n} \mid q_{i}=q_{j}\right\} .
$$

The set $\Omega_{n} \backslash \Delta$ is called the configuration space.

Definition 1. A configuration $q \in \Omega_{n} \backslash \Delta$ is called a central configuration (c.c.) if there is some constant $\lambda$ such that

$$
M^{-1} \frac{\partial U}{\partial q}=\lambda q .
$$

Equations (4) are invariant under rotation, dilatation and reflection on the plane. Two central configurations are considered equivalent if they are related by these symmetry operations.

The question of the existence and classification of central configuration is a fascinating problem that dates back to the 18th century. In 1767, Euler discovered the collinear c.c.'s. In 1772 Lagrange proved that, for any three arbitrary masses, the equilateral triangle is a central configuration.

For the collinear $n$-body problem an exact count of the central configurations of $n$ bodies was found by Moulton [14 (see also [15] for a modern proof). There is a unique collinear relative equilibrium for any ordering of the masses so there are $n ! / 2$ collinear equivalence classes.

The number of planar central configurations of the $n$-body problem for an arbitrary given set of positive masses, has been estabilished only for $n=3$ : there are always five relative equilibria. Two of these are Lagrange's equilateral triangles and the other three are collinear c.c. discovered by Euler. Already in the four body problem there is sufficient complexity to prevent a complete classification of the non-collinear relative equilibria. In fact, an exact count is known only for the equal masses case [1,2] and for certain cases where some of the masses are assumed sufficiently small 20 , 17.

Even the finiteness of the central configurations is a very difficult question. This conjecture was proposed by Chazy [7] and Wintner [19] and was listed by Smale as problem number 6 on his list of problems for this century [16. The finiteness problem was settled by A. Albouy [1,2] for the case of four equal masses and by Marshall Hampton and Rick Moeckel [9] for the general four body problem.

Aside from these fundamental results very little else is known in terms of the classification of c.c.'s for $n \geq 4$. One interesting open problem concerning the classification of c.c.'s, recently emphasized by A. Albouy and Y. Fu [6], 
is the following: Prove that, in the planar four-body problem, there is exactly one convex central configuration such that two given masses are not adjacent (i.e. they are on the same diagonal).

It is the scope of this paper to solve this conjecture in two particular cases. A first step in this direction was done by Y. Long and S. Sun [11. They proved that any convex non-collinear convex central configuration with masses $\delta>\alpha>0$, such that the diagonal corresponding to the mass $\alpha$ is not shorter than the one corresponding to the mass $\delta$, must posses a symmetry and therefore must be a kite. However, in their paper, they ask whether there are asymmetryc c.c.'s when the diagonal corresponding to the mass $\alpha$ is shorter than the other one. We show that this is not possible.

The main result of the paper is an extension of the above result, where we consider that only two of the masses are equal and at most, only one of the remaining masses is larger than the equal masses. We have the following

Theorem 1. Let $q=\left(q_{1}, q_{2}, q_{3}, q_{4}\right) \in \Omega_{4}$ be a convex non-collinear central configuration with masses $(\delta, \delta, \alpha, \beta) \in\left(\mathbb{R}^{+}\right)^{4}$. Suppose that the equal masses are opposite vertices and that $\alpha \leq \delta$ or $\beta \leq \delta$. Then the configuration $q$ must posses a symmetry, it is unique and forms a kite.

The uniqueness of the kite central configuration, in the hypothesis of the above theorem was proved by E. Leandro in 10 . We therefore have the following

Corollary 1. Under the hypothesis of Theorem 1 there is exactly one central configuration.

In particular, in the case $\alpha=\beta$ we prove the following

Theorem 2. Let $q=\left(q_{1}, q_{2}, q_{3}, q_{4}\right) \in \Omega_{4}$ be a convex non-collinear central configuration with masses $(\delta, \delta, \alpha, \alpha) \in\left(\mathbb{R}^{+}\right)^{4}$. Suppose that the equal masses are opposite vertices then the configuration $q$ must posses a symmetry and forms a rhombus.

This theorem completely answers the question of Y. Long and S. Sun 11. The uniqueness of the rhombus central configuration, in the hypothesis of the above theorem, is easy to prove (see for example [11 for a simple proof). We therefore have the following

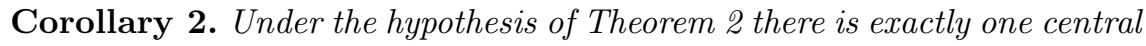
configuration.

In the next section we give some basic results and settings. In Section 3 we prove Theorem 1. In Section 4 we prove Theorem 2, In the Appendix we list different ways to write the equations of the balanced configurations of A. Albouy and A. Chenciner [5]. 


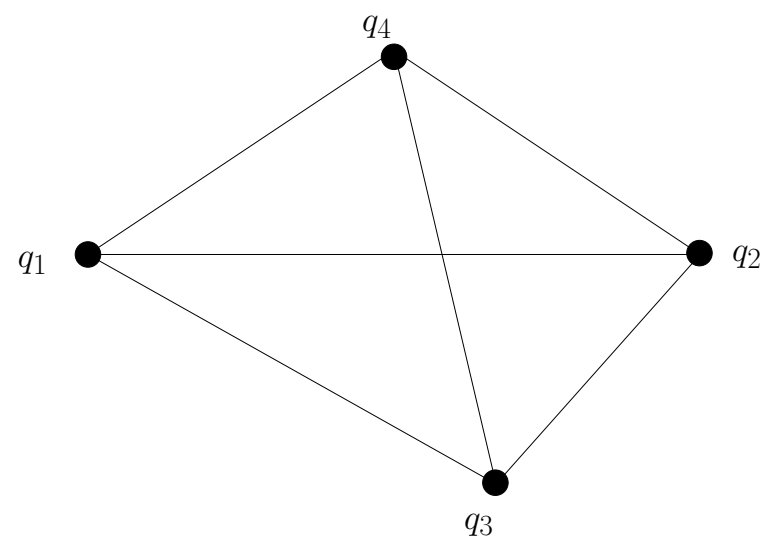

Fig. 1. A convex configuration of four masses.

\section{Acknowledgments}

The authors would like to thank A. Albouy and to an anonymous referee for their comments and suggestions regarding this work. Ernesto PérezChavela was supported by CONACYT México, grant no. 47768 .

\section{Preliminaries}

Firstly observe that if $\left(q_{1}, q_{2}, q_{3}, q_{4}\right) \in \Omega_{4}$ is a central configuration with parameter $\lambda$ and positive masses $\left(m_{1}, m_{2}, m_{3}, m_{4}\right)$ then, for every $\eta>0,\left(\frac{q_{1}}{\sqrt[3]{\eta}}, \frac{q_{2}}{\sqrt[3]{\eta}}, \frac{q_{4}}{\sqrt[3]{\eta}}, \frac{q_{4}}{\sqrt[3]{\eta}}\right) \in \Omega_{4}$ is the same central configuration with masses $\left(\frac{m_{1}}{\eta}, \frac{m_{2}}{\eta}, \frac{m_{3}}{\eta}, \frac{m_{4}}{\eta}\right)$ and the same value of $\lambda$. So, without loss of generality we suppose $\delta=1$, and we consider the planar 4-body problem with masses

$$
m_{1}=m_{2}=1, \quad m_{3}=\alpha, \quad m_{4}=\beta .
$$

In this paper we use Dziobeck coordinates, that we describe below (see 11] and [1] for more details). Let

$$
a=r_{12}^{2}, b=r_{13}^{2}, c=r_{14}^{2}, d=r_{23}^{2}, e=r_{24}^{2}, f=r_{34}^{2} .
$$

For $1 \leq i \leq 4$ let $\left|\Delta_{i}\right|$ be the area of the sub-triangle formed by the remaining three vertices of the configuration $q$ when deleting the point $q_{i}$. As in [11, we define the oriented areas of these sub-triangles of the convex non-collinear configuration $q$ by

$$
\Delta_{1}=-\left|\Delta_{1}\right|, \quad \Delta_{2}=-\left|\Delta_{2}\right|, \quad \Delta_{3}=\left|\Delta_{3}\right|, \quad \Delta_{4}=\left|\Delta_{4}\right|
$$

when the masses are opposite vertices of a quadrilateral. The $\Delta_{i}$ above satisfy the equation

$$
\Delta_{1}+\Delta_{2}+\Delta_{3}+\Delta_{4}=0
$$


The Cayley determinant

$$
S=\left|\begin{array}{lllll}
0 & 1 & 1 & 1 & 1 \\
1 & 0 & a & b & c \\
1 & a & 0 & d & e \\
1 & b & d & 0 & f \\
1 & c & d & f & 0
\end{array}\right|
$$

satisfies $S=0$. In 1900 Dziobek proved that

$$
\frac{\partial S}{\partial r_{i j}^{2}}=32 \Delta_{i} \Delta_{j} \quad \forall i \neq j .
$$

Let $\psi(s)=s^{-1 / 2}$ for $s>0$. Then the potential function and the moment of inertia are given by

$$
U=\sum_{1 \leq i<j \leq 4} m_{i} m_{j} \psi\left(r_{i j}^{2}\right)
$$

and

$$
I=\frac{1}{m^{\prime}} \sum_{1 \leq i<j \leq 4} m_{i} m_{j} r_{i j}^{2}
$$

respectively, where $m^{\prime}=\sum_{i=1}^{4} m_{i}$. Using Lagrange multipliers, Dziobek characterized the central configurations of four bodies as the extrema of

$$
U-\lambda S-\mu\left(I-I_{0}\right)
$$

as a function of $\lambda, \mu, r_{12}, \ldots, r_{34}$, where $\lambda$ and $\mu$ are Lagrange multipliers and $I_{0}$ is a fixed moment of inertia. Thus, for any $i, j$ with $1 \leq i<j \leq 4$, the central configurations satisfy

$$
\frac{\partial U}{\partial r_{i j}^{2}}=\lambda \frac{\partial S}{\partial r_{i j}^{2}}+\mu \frac{\partial I}{\partial r_{i j}^{2}},
$$

and from (8), we also have

$$
\frac{\partial U}{\partial r_{i j}^{2}}=m_{i} m_{j} \psi^{\prime}\left(r_{i j}^{2}\right)
$$

where $\psi^{\prime}(s)$ denotes the derivative of the function $\psi(s)$ with respect to $s$ and

$$
\frac{\partial I}{\partial r_{i j}^{2}}=\frac{m_{i} m_{j}}{m^{\prime}} .
$$

Consequently, equation (10) becomes

$$
m_{i} m_{j} \psi^{\prime}\left(r_{i j}^{2}\right)=32 \lambda \Delta_{i} \Delta_{j}+\frac{m_{i} m_{j} \mu}{m^{\prime}} .
$$


Therefore, using our mass convention, the equations for the central configurations are:

$$
\begin{aligned}
& \psi^{\prime}\left(r_{12}^{2}\right)=\nu \Delta_{1} \Delta_{2}+\xi \\
& \psi^{\prime}\left(r_{13}^{2}\right)=\frac{\nu}{\alpha} \Delta_{1} \Delta_{3}+\xi \\
& \psi^{\prime}\left(r_{14}^{2}\right)=\frac{\nu}{\beta} \Delta_{1} \Delta_{4}+\xi \\
& \psi^{\prime}\left(r_{23}^{2}\right)=\frac{\nu}{\alpha} \Delta_{2} \Delta_{3}+\xi \\
& \psi^{\prime}\left(r_{24}^{2}\right)=\frac{\nu}{\beta} \Delta_{2} \Delta_{4}+\xi \\
& \psi^{\prime}\left(r_{34}^{2}\right)=\frac{\nu}{\alpha \beta} \Delta_{3} \Delta_{4}+\xi
\end{aligned}
$$

where $\nu=32 \lambda$ and $\xi=\frac{\mu}{m^{\prime}}$. Moreover, there are implicit relations between the $r_{i j}^{2}$ and the $\Delta_{i}$ :

$$
t_{k}=\sum_{i=1}^{4} \Delta_{i} r_{i k}^{2}, \quad t_{1}=t_{2}=t_{3}=t_{4} .
$$

Using the implicit relations above we can prove the following two Lemmas due to A. Albouy [4]

Lemma 1. For a central configuration, the corresponding $\nu$ in the equations (12a)-12f] is positive.

Lemma 2. The following inequality holds:

$$
\left(\frac{\Delta_{i}}{m_{i}}-\frac{\Delta_{j}}{m_{j}}\right)\left(\Delta_{i}-\Delta_{j}\right) \geq 0 .
$$

Consequently $\Delta_{i}>\Delta_{j}$ if and only if $\frac{\Delta_{i}}{m_{i}}>\frac{\Delta_{j}}{m_{j}}$.

We now prove Lemma 1 and Lemma 2. From (13) we deduce

$$
0=t_{i}-t_{j}=r_{i j}^{2}\left(\Delta_{j}-\Delta_{i}\right)+\sum_{k} \Delta_{k}\left(r_{i k}^{2}-r_{j k}^{2}\right)
$$

and

$$
0=\left(\frac{\Delta_{i}}{m_{i}}-\frac{\Delta_{j}}{m_{j}}\right)\left(\Delta_{j}-\Delta_{i}\right) r_{i j}^{2}+m_{k} \sum_{k}\left(\frac{\Delta_{i} \Delta_{k}}{m_{i} m_{k}}-\frac{\Delta_{j} \Delta_{k}}{m_{j} m_{k}}\right)\left(r_{i k}^{2}-r_{j k}^{2}\right) .
$$

Using equation (11) we get

$$
0=\left(\frac{\Delta_{i}}{m_{i}}-\frac{\Delta_{j}}{m_{j}}\right)\left(\Delta_{j}-\Delta_{i}\right) r_{i j}^{2}+\frac{m_{k}}{32 \lambda} \sum_{k}\left(\psi^{\prime}\left(r_{i k}^{2}\right)-\psi^{\prime}\left(r_{j k}^{2}\right)\right)\left(r_{i k}^{2}-r_{j k}^{2}\right) .
$$

Since $\psi^{\prime}(s)$ is a monotone increasing function of $s\left(\psi^{\prime}\left(r_{i k}^{2}\right)-\psi^{\prime}\left(r_{j k}^{2}\right)\right)\left(r_{i k}^{2}-\right.$ $\left.r_{j k}^{2}\right) \geq 0$. Thus

$$
\lambda\left(\frac{\Delta_{i}}{m_{i}}-\frac{\Delta_{j}}{m_{j}}\right)\left(\Delta_{i}-\Delta_{j}\right) \geq 0
$$


Let us choose the index $i$ corresponding to the smallest $\Delta_{i}$, and $j$ corresponding to the greatest $\Delta_{j}$. We have $\Delta_{i}<0<\Delta_{j}$, because $\sum \Delta_{k}=0$. Moreover if $\lambda=0$ all the edges are equal, but this is geometrically impossible, thus $\lambda>0, \nu>0$. This concludes Lemma 1 Moreover

$$
\left(\frac{\Delta_{i}}{m_{i}}-\frac{\Delta_{j}}{m_{j}}\right)\left(\Delta_{i}-\Delta_{j}\right) \geq 0 . \quad \text { for any } i, j
$$

this concludes the proof of Lemma 2

Let

$$
A=\psi^{\prime}(a), B=\psi^{\prime}(b), \ldots, F=\psi^{\prime}(f) .
$$

From (13) one can extract some weaker identities

$$
Q_{i j k}=\left|\begin{array}{ccc}
1 & 1 & 1 \\
t_{i} & t_{j} & t_{k} \\
\frac{\Delta_{i}}{m_{i}} & \frac{\Delta_{j}}{m_{j}} & \frac{\Delta_{k}}{m_{k}}
\end{array}\right|=0 .
$$

Of course $Q_{i j k}=0$ if $t_{i}=t_{j}=t_{k}$. But if all the $Q_{i j k}$ are zero one can only deduce that $t_{i}=\eta \Delta_{i} / m_{i}+\delta$ for some $(\eta, \delta) \in \mathbb{R}^{2}$ and for all $i$. For the four body problem equations (16), using the fact that $\Delta_{1}+\Delta_{2}+\Delta_{3}+\Delta_{4}=0$, after some tedious computations we obtain

$$
\begin{aligned}
& \left|\begin{array}{ccc}
1 & 1 & 1 \\
f-e-d & \alpha(e-d-f) & \beta(d-f-e) \\
F & E & D
\end{array}\right|=\left|\begin{array}{ccc}
1 & 1 & 1 \\
a+f & b+e & c+d \\
A & B & C
\end{array}\right| \\
& \left|\begin{array}{ccc}
1 & 1 & 1 \\
f-c-b & \beta(b-f-c) & \alpha(c-b-f) \\
F & B & C
\end{array}\right|=\left|\begin{array}{ccc}
1 & 1 & 1 \\
a+f & b+e & c+d \\
A & E & D
\end{array}\right| \\
& \left|\begin{array}{ccc}
1 & 1 & 1 \\
\beta(a-e-e) & e-c-a & c-a-e \\
A & E & C
\end{array}\right|=\alpha\left|\begin{array}{ccc}
1 & 1 & 1 \\
a+f & b+e & c+d \\
F & B & D
\end{array}\right| \\
& \left|\begin{array}{ccc}
1 & 1 & 1 \\
\alpha(a-d-b) & b-a-d & d-b-a \\
A & B & D
\end{array}\right|=\beta\left|\begin{array}{ccc}
1 & 1 & 1 \\
a+f & b+e & c+d \\
F & E & C
\end{array}\right|
\end{aligned}
$$

These are the equations of the balanced configurations (configuration équilibrée) due to A. Albouy and A. Chenciner [5]. In the Appendix we present other ways to write the above identities.

Observe that the determinant

$$
d(u, v, w ; U, V, W)=\left|\begin{array}{ccc}
1 & 1 & 1 \\
u & v & w \\
U & V & W
\end{array}\right|
$$

has a beautiful geometrical interpretation. In fact $d(u, v, w ; U, V, W)$ is the oriented area of the triangle of vertices $(u, U),(v, V),(w, W)$ (see [18]) where the sign is determined by the following Lemma: 
Lemma 3. Let

$$
V^{\prime}=\frac{v-w}{u-w} U+\frac{u-v}{u-w} W
$$

1. the following holds:

$$
d(u, v, w ; U, V, W)=(u-w)\left(V-V^{\prime}\right) .
$$

2. The determinant $d(u, v, w ; U, V, W)>0$ provided $u>v>w$ and $V>$ $V^{\prime}$, i.e. the point $(v, V)$ is located strictly above the line passing through $(w, W)$ and $(u, U)$.

3. The determinant $d(u, v, w ; U, V, W)<0$ provided $u>v>w$ and $V<$ $V^{\prime}$, i.e. the point $(v, V)$ is located strictly below the line passing through $(w, W)$ and $(u, U)$.

4. Let $g:(0,+\infty) \rightarrow(0, \infty)$ be a strictly concave function. Suppose $u>$ $v>w$. Then $d(u, v, w, g(u), g(v), g(w))>0$.

Lemma 3 will be useful in proving the main results of this paper. A proof can be found in [11.

\section{Proof of Theorem 1}

Observe that, in order to have symmetry, under the hypotesis of Theorem 1 the following inequality must hold

$$
\Delta_{1}=\Delta_{2}
$$

Note that $\Delta_{3} / \alpha=\Delta_{4} / \beta$ or $\Delta_{3}=\Delta_{4}$ only if one is in a symmetric configurations with $\alpha=\beta$ (see [3,4 for more details). In this section we assume $\beta \neq \alpha$. To show that a configuration is symmetric, i.e. that (22) holds, one can assume that

$$
\Delta_{1} \neq \Delta_{2} \quad \text { and } \quad \Delta_{3} \neq \Delta_{4}
$$

and then derive a contradiction. This is the strategy of the proof. If we assume $\Delta_{1} \neq \Delta_{2}$ and $\Delta_{3} \neq \Delta_{4}$ we have four cases

(a) $\Delta_{1}<\Delta_{2}<0<\Delta_{3}<\Delta_{4}$
(b) $\Delta_{2}<\Delta_{1}<0<\Delta_{3}<\Delta_{4}$
(c) $\Delta_{1}<\Delta_{2}<0<\Delta_{4}<\Delta_{3}$
(d) $\Delta_{2}<\Delta_{1}<0<\Delta_{4}<\Delta_{3}$.

Moreover the mutual distances satisfy some geometrical inequalities. We have then 
Lemma 4. The following inequalities hold

$$
\begin{aligned}
& c<\min \{b, e\} \leq \max \{b, e\}<d<\min \{a, f\} \leq \max \{a, f\} \\
& e<\min \{c, d\} \leq \max \{c, d\}<b<\min \{a, f\} \leq \max \{a, f\} \\
& b<\min \{c, d\} \leq \max \{c, d\}<e<\min \{a, f\} \leq \max \{a, f\} \\
& d<\min \{b, e\} \leq \max \{b, e\}<c<\min \{a, f\} \leq \max \{a, f\}
\end{aligned}
$$

in the cases a,b,c and $d$ respectively.

Proof. We prove (24a), the other cases are similar. Case (a) has 3 possible subcases.

- Subcase 1. $\Delta_{1}+\Delta_{4}=0$. In this case, by equation (5) we obtain

$$
\Delta_{1}=-\Delta_{4}, \quad \Delta_{2}=-\Delta_{3} .
$$

Thus we have

$$
\Delta_{1} \Delta_{4}<\Delta_{1} \Delta_{3}=\Delta_{2} \Delta_{4}<\Delta_{2} \Delta_{3}<0<\Delta_{1} \Delta_{2}=\Delta_{3} \Delta_{4} .
$$

Moreover by Lemma 1 and Lemma 2 we have

$$
\begin{aligned}
\frac{\nu}{\beta} \Delta_{1} \Delta_{4}<\min \left\{\frac{\nu}{\alpha} \Delta_{1} \Delta_{3}\right. & \left., \frac{\nu}{\beta} \Delta_{2} \Delta_{4}\right\} \\
<\frac{\nu}{\alpha} \Delta_{2} \Delta_{3} & \left.<0<\nu \Delta_{1} \Delta_{2}<\frac{\nu}{\alpha \beta} \Delta_{3} \Delta_{4}, \frac{\nu}{\beta} \Delta_{2} \Delta_{4}\right\}
\end{aligned}
$$

Therefore by equations (12a)-(12f) and the monotonicity of $\psi^{\prime}(s)$ we have

$$
c<\min \{b, e\} \leq \max \{b, e\}<d<a<f .
$$

- Subcase 2. $\Delta_{1}+\Delta_{4}<0$.

In this case, by equation (5) we have $\Delta_{2}+\Delta_{3}>0$. Using equation (5) yields

$$
\begin{aligned}
\Delta_{2} \Delta_{4}-\Delta_{1} \Delta_{3}= & \Delta_{2} \Delta_{4}+\Delta_{1}\left(\Delta_{1}+\Delta_{2}+\Delta_{4}\right) \\
& =\left(\Delta_{2}+\Delta_{1}\right)\left(\Delta_{4}+\Delta_{1}\right)>0 .
\end{aligned}
$$

Similarly

$$
\begin{aligned}
\Delta_{3} \Delta_{4}-\Delta_{1} \Delta_{2} & =\Delta_{3} \Delta_{4}+\Delta_{1}\left(\Delta_{2}+\Delta_{3}+\Delta_{4}\right) \\
& =\left(\Delta_{1}+\Delta_{3}\right)\left(\Delta_{1}+\Delta_{4}\right)>0
\end{aligned}
$$

since $\Delta_{1}+\Delta_{4}<0$ together with $\Delta_{1}<\Delta_{2}<0<\Delta_{3}<\Delta_{4}$ implies $\Delta_{1}+\Delta_{3}<0$. Consequently we have

$$
\Delta_{1} \Delta_{4}<\Delta_{1} \Delta_{3}<\Delta_{2} \Delta_{4}<\Delta_{2} \Delta_{3}<0<\Delta_{1} \Delta_{2}<\Delta_{3} \Delta_{4}
$$


and using Lemma 1 and Lemma 2 we obtain

$$
\begin{aligned}
\frac{\nu}{\beta} \Delta_{1} \Delta_{4}<\min \left\{\frac{\nu}{\alpha} \Delta_{1} \Delta_{3}\right. & \left., \frac{\nu}{\beta} \Delta_{2} \Delta_{4}\right\} \\
<\frac{\nu}{\alpha} \Delta_{2} \Delta_{3} & <0<\nu \Delta_{1} \Delta_{2}<\frac{\nu}{\alpha \beta} \Delta_{3} \Delta_{4} .
\end{aligned}
$$

Thus, as in Subcase 1, we get

$$
c<\min \{b, e\} \leq \max \{b, e\}<d<a<f .
$$

- Subcase 3: $\Delta_{1}+\Delta_{4}>0$.

In this case equation (5) implies that $\Delta_{2}+\Delta_{3}<0$. Hence

$$
\Delta_{2} \Delta_{4}-\Delta_{1} \Delta_{3}=\left(\Delta_{2}+\Delta_{1}\right)\left(\Delta_{4}+\Delta_{1}\right)<0
$$

and

$$
\Delta_{1} \Delta_{4}<\Delta_{2} \Delta_{4}<\Delta_{1} \Delta_{3}<\Delta_{2} \Delta_{3} .
$$

Using Lemma 1 and Lemma 2 we find

$$
\begin{aligned}
\frac{\nu}{\beta} \Delta_{1} \Delta_{4}<\min \left\{\frac{\nu}{\alpha} \Delta_{1} \Delta_{3}, \frac{\nu}{\beta} \Delta_{2} \Delta_{4}\right\} & \leq \max \left\{\frac{\nu}{\alpha} \Delta_{1} \Delta_{3}, \frac{\nu}{\beta} \Delta_{2} \Delta_{4}\right\} \\
& <\frac{\nu}{\alpha} \Delta_{2} \Delta_{3}<0<\nu \Delta_{1} \Delta_{2}
\end{aligned}
$$

and thus

$$
c<\min \{b, e\} \leq \max \{b, e\}<d<\min \{a, f\} \leq \max \{a, f\} .
$$

Therefore, in all three subcases inequality (24a) holds. This concludes the proof of the Lemma.

We can now continue the proof of Theorem 1. From (20), using the fundamental properties of the determinants, we deduce

$$
(\alpha+1)\left|\begin{array}{lll}
1 & 1 & 1 \\
a & b & d \\
A & B & D
\end{array}\right|-(\alpha-1)[A(d-b)+b B-d D]=\beta\left|\begin{array}{lll}
1 & 1 & 1 \\
f & e & c \\
F & E & C
\end{array}\right|+\beta\left|\begin{array}{lll}
1 & 1 & 1 \\
a & b & d \\
F & E & C
\end{array}\right| .
$$

Now consider the cases (a) and (c). Then, by equation (24a) and (24c), Lemma 3 (2) and the concavity of the function $\psi^{\prime}(s)$, we obtain

$$
\left|\begin{array}{lll}
1 & 1 & 1 \\
a & b & d \\
A & B & D
\end{array}\right|<0, \quad\left|\begin{array}{lll}
1 & 1 & 1 \\
f & e & c \\
F & E & C
\end{array}\right|>0, \quad\left|\begin{array}{lll}
1 & 1 & 1 \\
a & b & d \\
F & E & C
\end{array}\right|>0
$$

We also have that $d-b>0, A<0$ and $b B-d D<0$ since

$$
b B-d D=\frac{1}{2}\left(\frac{\sqrt{b}-\sqrt{d}}{\sqrt{b d}}\right)<0 .
$$


Consequently $-(\alpha-1)[A(d-b)+b B-d D]<0$ when $0<\alpha \leq 1$ and the left hand side of (28) is negative. This produces a contradiction since the right hand side of (28) is positive for any value of $\beta>0$.

In the cases (b) and (d) we have

$$
\left|\begin{array}{lll}
1 & 1 & 1 \\
a & b & d \\
A & B & D
\end{array}\right|>0, \quad\left|\begin{array}{lll}
1 & 1 & 1 \\
f & e & c \\
F & E & C
\end{array}\right|<0, \quad\left|\begin{array}{ccc}
1 & 1 & 1 \\
a & b & d \\
F & E & C
\end{array}\right|<0 .
$$

We also have that $d-b<0, A<0$ and $b B-d D>0$.

Consequently $-(\alpha-1)[A(d-b)+b B-d D]>0$ when $0<\alpha \leq 1$ and the left hand side of (28) is positive for any value of $\beta>0$. This produces a contradiction since the right hand side of (28) is negative.

From (19), using the fundamental properties of the determinants we deduce

$$
(\beta+1)\left|\begin{array}{lll}
1 & 1 & 1 \\
a & e & c \\
A & E & C
\end{array}\right|-(\beta-1)[A(c-e)+e E-c C]=\alpha\left|\begin{array}{lll}
1 & 1 & 1 \\
a & e & c \\
F & B & D
\end{array}\right|+\alpha\left|\begin{array}{lll}
1 & 1 & 1 \\
f & b & d \\
F & B & D
\end{array}\right| .
$$

Now consider the cases (a) and (c). Then we find

$$
\left|\begin{array}{lll}
1 & 1 & 1 \\
a & e & c \\
A & E & C
\end{array}\right|>0, \quad\left|\begin{array}{lll}
1 & 1 & 1 \\
a & e & c \\
F & B & D
\end{array}\right|<0, \quad\left|\begin{array}{ccc}
1 & 1 & 1 \\
f & b & d \\
F & B & D
\end{array}\right|<0
$$

We also have that $c-e<0, A<0$ and $e E-c C>0$ since

$$
e E-c C=\frac{1}{2}\left(\frac{\sqrt{e}-\sqrt{c}}{\sqrt{e c}}\right)>0 .
$$

Consequently $-(\beta-1)[A(c-e)+e E-c C]>0$ when $0<\beta \leq 1$ and the left hand side of (28) is negative for any value of $\alpha>0$. This produces a contradiction since the right hand side of (28) is positive.

In the cases (b) and (d) we have

$$
\left|\begin{array}{lll}
1 & 1 & 1 \\
a & e & c \\
A & E & C
\end{array}\right|<0, \quad\left|\begin{array}{lll}
1 & 1 & 1 \\
a & e & c \\
F & B & D
\end{array}\right|>0, \quad\left|\begin{array}{ccc}
1 & 1 & 1 \\
f & b & d \\
F & B & D
\end{array}\right|>0
$$

We also have that $c-e>0, A<0$ and $e E-c C<0$.

Consequently $-(\beta-1)[A(c-e)+e E-c C]>0$ when $0<\beta \leq 1$ and the left hand side of (31) is negative for any value of $\alpha>0$. This produces a contradiction since the right hand side of (31) is positive.

This proves that $\Delta_{1}=\Delta_{2}$ and thus it shows the existence of a line of symmetry.

We now show that the configuration must be a kite

Lemma 5. If $\Delta_{1}=\Delta_{2}$ the quadrilateral $q$ is a kite. 
Proof. By equations (12a, 12f) of the central configurations we have

$$
\psi^{\prime}\left(r_{13}^{2}\right)=\frac{\nu}{\alpha} \Delta_{1} \Delta_{3}+\xi=\frac{\nu}{\alpha} \Delta_{2} \Delta_{3}+\xi=\psi^{\prime}\left(r_{23}^{2}\right) .
$$

Since $\psi^{\prime}(s)$ is a monotone increasing function of $s$ we obtain

$$
r_{13}=r_{23} .
$$

Similarly

$$
\psi^{\prime}\left(r_{14}^{2}\right)=\frac{\nu}{\beta} \Delta_{1} \Delta_{4}+\xi=\frac{\nu}{\beta} \Delta_{2} \Delta_{4}+\xi=\psi^{\prime}\left(r_{24}^{2}\right) .
$$

and thus

$$
r_{14}=r_{24} .
$$

Therefore the quadrilateral is a kite.

This concludes the proof of Theorem 1

\section{Proof of Theorem 2}

In this case $\beta=\alpha$. Observe that, in order to prove Theorem 2 it is enough to study the case $0<\alpha \leq \delta=1$. In fact the case $\alpha \geq \delta=1$ can be obtained from the previous one just renaming the masses).

In order to prove the existence of a line of symmetry, in the configuration under consideration in this paper, we need to have either $\Delta_{1}=\Delta_{2}$ or $\Delta_{3}=\Delta_{4}$. As in Theorem 1 we can assume that $\Delta_{1} \neq \Delta_{2}$ and $\Delta_{3} \neq \Delta_{4}$ and prove the existence of the line of symmetry by contradiction. Even in this case one obtain the four cases in equation (23) and Lemma 4 holds.

The reminder of the proof follows directly from the one of Theorem 1 with $\beta=\alpha$, since as observed above one needs only to consider the case $\alpha \geq 1$.

The main reason to include this result as a Theorem is on one hand, that it completely answers the question formulated by Y. Long and S. Sun in [11, and on the other hand, that is not obvious from the equations of the balanced configurations, or from Dziobek equations that the kite central configuration obtained using Theorem 1 is a rhombus, but it follows from the uniqueness of the rhombus central configuration and a result by E. Leandro 10] that can be summarized as follows

Lemma 6. For any $\alpha>0$ and $\beta>0$, there exists a unique central configuration $q=\left(q_{1}, q_{2}, q_{3}, q_{4}\right)$ with masses $(1,1, \alpha, \beta)$ where $q_{1}$ and $q_{2}$ as well as $q_{3}$ and $q_{4}$ are located at the opposite vertices of a kite shaped quadrilateral.

This concludes the proof of Theorem 2 . 


\section{Appendix}

From (17][20), using the fundamental properties of the determinants we deduce the following identities

$$
\begin{gathered}
-(1-\alpha)(f-e-d)(D-E)+(\beta-\alpha)(d-f-e)(F-E)+2 \alpha\left|\begin{array}{lll}
1 & 1 & 1 \\
f & e & d \\
F & E & D
\end{array}\right| \\
=\left|\begin{array}{lll}
1 & 1 & 1 \\
a & b & c \\
A & B & C
\end{array}\right|+\left|\begin{array}{lll}
1 & 1 & 1 \\
f & e & d \\
A & B & C
\end{array}\right| . \\
-(1-\alpha)(f-c-b)(C-B)+(\beta-\alpha)(b-f-c)(C-F)+2 \alpha\left|\begin{array}{lll}
1 & 1 & 1 \\
f & b & c \\
F & B & C
\end{array}\right| \\
=\left|\begin{array}{lll}
1 & 1 & 1 \\
a & e & d \\
A & E & D
\end{array}\right|+\left|\begin{array}{lll}
1 & 1 & 1 \\
f & b & c \\
A & E & D
\end{array}\right| . \\
-(\beta-1)(a-e-c)(C-E)+2\left|\begin{array}{lll}
1 & 1 & 1 \\
a & e & c \\
A & E & C
\end{array}\right|=\alpha\left|\begin{array}{lll}
1 & 1 & 1 \\
a & e & c \\
F & B & D
\end{array}\right|+\alpha\left|\begin{array}{lll}
1 & 1 & 1 \\
f & b & d \\
F & B & D
\end{array}\right|,(40) \\
-(\alpha-1)(a-d-b)(D-B)+2\left|\begin{array}{lll}
1 & 1 & 1 \\
a & b & d \\
A & B & D
\end{array}\right|=\beta\left|\begin{array}{lll}
1 & 1 & 1 \\
f & e & c \\
F & E & C
\end{array}\right|+\beta\left|\begin{array}{lll}
1 & 1 & 1 \\
a & b & d \\
F & E & C
\end{array}\right| .(41)
\end{gathered}
$$

Note that if $\beta=\alpha$ the identities above reduce to the expression of the balanced configurations used in [11.

The equation of the balanced configurations can also be written in a different way, that seems to be useful in certain problems (e.g. to prove the main result of this paper, Theorem [1). From (17,20) we find

$$
\begin{aligned}
(1+\beta) & \left|\begin{array}{lll}
1 & 1 & 1 \\
f & e & d \\
F & E & D
\end{array}\right|-(1-\alpha)[F(d-e)+e E-d D]-(\beta-\alpha)[D(e-f)+f F-e E] \\
& =\left|\begin{array}{lll}
1 & 1 & 1 \\
a & b & c \\
A & B & C
\end{array}\right|+\left|\begin{array}{lll}
1 & 1 & 1 \\
f & e & d \\
A & B & C
\end{array}\right|
\end{aligned}
$$




$$
\begin{aligned}
(\beta+1) & \left|\begin{array}{lll}
1 & 1 & 1 \\
f & b & c \\
F & B & C
\end{array}\right|-(1-\alpha)[F(c-b)+b B-c C]-(\beta-\alpha)[B(f-c)+c C-f F] \\
& =\left|\begin{array}{lll}
1 & 1 & 1 \\
a & e & d \\
A & E & D
\end{array}\right|+\left|\begin{array}{lll}
1 & 1 & 1 \\
f & b & c \\
A & E & D
\end{array}\right|
\end{aligned}
$$

$$
\begin{aligned}
& (\beta+1)\left|\begin{array}{lll}
1 & 1 & 1 \\
a & e & c \\
A & E & C
\end{array}\right|-(\beta-1)[A(c-e)+e E-c C]=\alpha\left|\begin{array}{lll}
1 & 1 & 1 \\
a & e & c \\
F & B & D
\end{array}\right|+\alpha\left|\begin{array}{lll}
1 & 1 & 1 \\
f & b & d \\
F & B & D
\end{array}\right|, \\
& (\alpha+1)\left|\begin{array}{lll}
1 & 1 & 1 \\
a & b & d \\
A & B & D
\end{array}\right|-(\alpha-1)[A(d-b)+b B-d D]=\beta\left|\begin{array}{lll}
1 & 1 & 1 \\
f & e & c \\
F & E & C
\end{array}\right|+\beta\left|\begin{array}{lll}
1 & 1 & 1 \\
a & b & d \\
F & E & C
\end{array}\right| .
\end{aligned}
$$

\section{References}

1. Albouy A.: Symétrie des configurations centrales de quatre corps. C.R. Acad. Sci Paris. 320, 217-220, (1995).

2. Albouy A.: The Symmetric Central Configurations of Four Equal Masses, Contemp. Math. V 198, 131-135, (1996)

3. Albouy A.: On a Paper of Moeckel on Central Configurations. Reg. Chaotic Dynamics 8, 133-142, (2003)

4. Albouy A.: Mutual Distances in Celestial Mechanics. preprint (2004)

5. Albouy A., Chenciner A.: Le probléme des $n$ corps et les distances mutuells. Invent. Mat. 131, 151-184, (1988)

6. Albouy A. and Fu Y.: Euler Configurations and Quasi-Polynomial Systems. preprint (2006)

7. Chazy J.: Sur Certaines trajectoires du probléme des n corps. Bull. Astron. 35, 321-389, (1918)

8. Hagihara Y. Celestial Mechanics, Vol. 1. MIT press, Massachusetts, 1970

9. Hampton M.: Moeckel R., Finiteness of Relative Equilibria of the FourBody Problem, Invent. math. 163, 289-312, (2006)

10. Leandro E.S.G.: Finitness and Bifurcations of some Symmetrical Classes of Central Configurations. Arch.Rational Mech Anal. 167, 147-177, (2003)

11. Long Y., Sun. S.: Four-Body Central Configurations with some Equal Masses. Arch. Rational Mech Anal. 162, 24-44, (2002)

12. MacMillan W., Bartky W.: Permanent Central Configurations in the Problem of Four Bodies. Trans Amer. Math. Soc. 34, 838-875, (1932)

13. Moeckel R.: On Central Configurations. Math Z., 205,499-517, (1990)

14. Moulton F.R.: The Straight Line Solutions of the Problem of $n$-bodies. Ann. Math. 12, 1-17, (1910)

15. Smale S.: Topology and Mechanics II. Inv. Math, 11, 45-64, (1970)

16. Smale S.: Mathematical Problems for the Next Century. Math. Intell., 20, 7-15, (1998)

17. Tien F.: Recursion Formulas of Central Configurations, Thesis, University of Minnesota, 1993 
18. Uspensky J.V.: Theory of Equations. McGraw Hill, New York, 1948

19. Wintner A.: The Analytical Foundations of Celestial Mechanics. Princeton Math. Series 5. Princeton University Press, Princeton NJ, 1941

20. Xia, Z.: Central Configurations with Many Small Masses. J. Diff. Equations, 91, 168-179, (1991)

21. Xia Z.: Convex Central Configurations for the $n$-body Problem. J. Diff. Eqn., 200,185-190, (2004)

Departamento de Matemáticas

UAM-Iztapalapa, A.P. 55-534

09340 Iztapalapa, Mexico, D.F., Mexico

email: epc@xanum.uam.mx

and

Department of Mathematics

Wilfrid Laurier University

75 University Avenue West, Waterloo, Canada

email: msantoprete@wlu.ca 